\title{
Liquid Helix: How Capillary Jets Adhere to Vertical Cylinders
}

\author{
E. Jambon-Puillet, ${ }^{1}$ W. Bouwhuis, ${ }^{2,3}$ J. H. Snoeijer, ${ }^{2}$ and D. Bonn ${ }^{1}$ \\ ${ }^{1}$ Institute of Physics, Van der Waals-Zeeman Institute, University of Amsterdam, Science Park 904, 1098 XH Amsterdam, Netherlands \\ ${ }^{2}$ Physics of Fluids Group, Faculty of Science and Technology, MESA+Institute, University of Twente, 7500 AE Enschede, Netherlands \\ ${ }^{3}$ School of Life Science, Engineering \& Design at Saxion University of Applied Sciences, P.O. Box 70.000, \\ Enschede $7500 \mathrm{~KB}$, Netherlands
}

(Received 27 November 2018; revised manuscript received 20 February 2019; published 8 May 2019)

\begin{abstract}
From everyday experience, we all know that a solid edge can deflect a liquid flowing over it significantly, up to the point where the liquid completely sticks to the solid. Although important in pouring, printing, and extrusion processes, there is no predictive model of this so-called "teapot effect." By grazing vertical cylinders with inclined capillary liquid jets, here we use the teapot effect to attach the jet to the solid and form a new structure: the liquid helix. Using mass and momentum conservation along the liquid stream, we first quantitatively predict the shape of the helix and then provide a parameter-free inertial-capillary adhesion model for the jet deflection and critical velocity for helix formation.
\end{abstract}

DOI: 10.1103/PhysRevLett.122.184501

When a liquid is poured too slowly from a container, it has the tendency to "stick" to the container edge, running down along the container's wall instead of separating from it. To avoid this inconvenience often referred to as the "teapot effect," millennia of empirical evidence has taught potters the design of the container edge, and in particular its sharpness, is of paramount importance. It was however demonstrated only recently by Duez et al. [1] that even for rapid inertial flows, the wettability of the surface also plays an unexpectedly important role and can be used to control liquid flow separation $[2,3]$. Yet, although the teapot effect has received attention from physicists for centuries [1-13], a simple quantitative description fully capturing the observations is still lacking. From a practical point of view, understanding the teapot effect is of paramount importance not only for designing food containers but also to better control flows through orifices [14], to avoid fouling up the nozzle of inkjet and 3D printers [15], and for polymer extrusion processes where capillary adhesion causes "sharkskin" instabilities [16].

In this Letter, we experimentally investigate the adhesion of capillary water jets to vertical cylinders (Fig. 1). Highspeed jets are deflected due to inertial-capillary adhesion, and upon decreasing the flow rate, they eventually fold around the cylinder and completely stick to it. The jet then turns into a steady rivulet which flows down the cylinder, forming an elegant novel fluidic structure, the liquid helix, thus, transforming an everyday annoyance into an simple way to produce complex patterns analogous to those of "liquid rope coiling" that recently received much attention [17]. We first investigate the rivulet helical trajectory over a wide range of geometrical parameters. We then look into the high-velocity regime when the jet is bent by the cylinder but still separates and identify the critical velocity to form a liquid helix. All these results can be accounted for using momentum conservation on the liquid stream, both for the helix shape and the jet deflection. In particular, the proposed model is the first to actually predict a sticking transition, and its scaling laws are in excellent agreement with experiments.

Our helix experiment is shown schematically in Fig. 1(a). A jet inclined by an angle $\psi_{0}$ with respect to the vertical is generated by flowing water (density $\rho=1 \mathrm{~g} / \mathrm{cm}^{3}$, viscosity $\eta=1 \mathrm{cP}$, surface tension $\gamma=72 \mathrm{mN} / \mathrm{m}$ ) from a pressurized tank through a nozzle [bore diameter $\left.0.2<D_{j}(\mathrm{~mm})<1.5\right]$. The volumetric flow rate $Q$ is kept constant (controlled by a precision valve and measured using a flow meter). The jet is impacted on a vertical cylinder made of glass (contact angles $\theta \approx 30^{\circ}$ with fluctuations between cylinders) or teflon $\left(\theta \approx 90^{\circ}\right)$ with a diameter $1.05<$ $D_{c}(\mathrm{~mm})<14.4$ (see Supplemental Material [18]). As the degree of overlap between the jet and the cylinder is a critical parameter $[11,12]$, we use a linear stage to translate the nozzle until it barely touches the cylinder. Figure 1(b) shows photographs of an experiment in which the flow rate is decreased and increased again. The pictures show that as the flow rate $Q$ is decreased, the water jet is increasingly bent by the glass cylinder until at a critical flow rate it completely sticks to the cylinder, forming a helical rivulet. This sticking transition is hysteretic: Increasing the flow rate again does not cause the immediate breakdown of the helix. For all our experiments, the jet Reynolds and Froude numbers are quite high: $360<\operatorname{Re}=\rho U_{0} D_{j} / \eta<6600$ and $7<\mathrm{Fr}=$ $U_{0} / \sqrt{g D_{j}}<308$ with $U_{0}=4 Q / \pi D_{j}^{2}$ the initial jet speed. The initial phase of the jet sticking will thus be governed by inertia, though it will turn out that viscosity and gravity affect the helix after a couple of revolutions. 


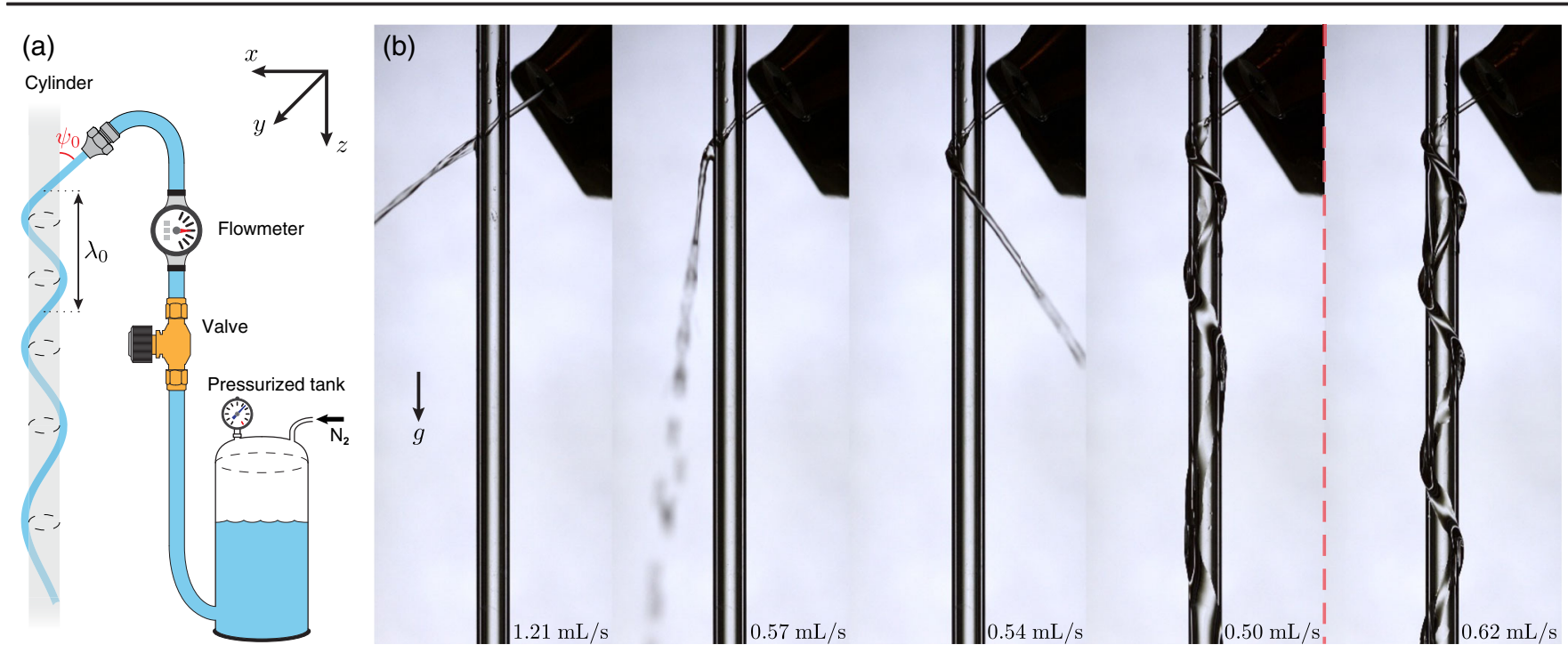

FIG. 1. (a) Schematic of the experiment indicating the initial inclination angle $\psi_{0}$ and helical pitch $\lambda_{0}$. (b) Side-view pictures of a sequence of experiments showing the deviation of a $0.5 \mathrm{~mm}$ water jet grazing a $3.0 \mathrm{~mm}$ glass cylinder. The flow rate $Q$ is decreased until the penultimate image and then increased again to illustrate the hysteresis in the sticking transition.

The shape of the helix.-We first focus on the helical rivulet regime, for which the jet sticks completely to the cylinder. Neglecting the small thickness variations, we assume that the fluid stream describes a helical motion with a constant helix radius $\mathcal{R}_{h}=\left(D_{c}+D_{j}\right) / 2$. The rivulet trajectory can then be parametrized by the rivulet arc length $s$ and its local angle with respect to the vertical $\psi(s)$. It is insightful to "unwrap" the trajectory on an effective Cartesian plane $(X, z)$ shown in Fig. 2(a). The azimuth $\phi$ is then replaced by $X=\mathcal{R}_{h} \phi$, and the tangent vector becomes planar (see Supplemental Material [18]). The problem then becomes mathematically equivalent to finding the trajectory of the rivulet formed by the impact of a jet of vanishing incidence on a flat plate. Given the large $\mathrm{Re}$ and $\mathrm{Fr}$, we anticipate the initial revolution to be dominated by inertia. The initial $z$ momentum is unchanged, while the $x$ momentum is transferred to the orthoradial direction $X$ (once unwrapped). Without viscous friction or gravity, the unwrapped rivulet trajectory is trivially a straight line; this corresponds to a helix of constant pitch $\lambda=2 \pi \mathcal{R}_{h} / \tan \psi_{0}$ once wrapped around the cylinder. In the inset of Fig. 2(a), we compare this prediction to the experimentally observed initial pitch $\lambda_{0}$ [defined in Fig. 1(a)]. Indeed, the inertial prediction accurately describes the initial pitch $\lambda_{0}$, except for the slowest jets.

However, the actual pitch is clearly not constant and increases as the helix goes down (Figs. 1 and 3). After a few turns, the rivulet has lost most of its orthoradial momentum, and the liquid only flows downward. Introducing gravity into the inertial description indeed stretches the helix but only by a negligible amount [see Fig. 2(b), blue curve]. Instead, a quantitative description of the helix calls for both gravity and viscous friction. In the spirit of the analysis of hydraulic jumps [26,27] and meandering rivulets [28,29], we therefore perform a momentum balance on an infinitesimal portion of the rivulet [see Fig. 2(a)] including gravity, viscous friction, and the inertial-capillary adhesion force. At steady state, the flux $Q=A U$ is constant along the helix, where we introduced $U(s)$ as the mean rivulet velocity averaged over the cross-sectional area $A(s)$. If we further introduce unit vectors along the rivulet, $\hat{\mathbf{t}}(s)$, and normal to the cylinder $\hat{\mathbf{n}}(s)$, the steady momentum balance reads
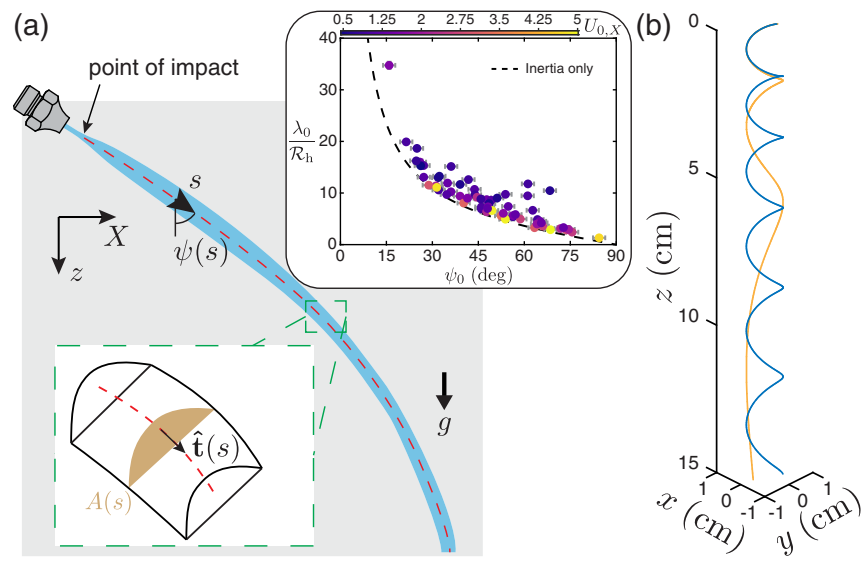

FIG. 2. (a) Sketch of the (unwrapped) rivulet model. The impacting jet turns into a rivulet whose centerline (drawn as a red dashed line) is parametrized by the arc length $s$ and the angle with respect to the vertical axis $\psi(s)$. Inset: Initial helix pitch $\lambda_{0} / \mathcal{R}_{h}$ as a function of the jet inclination angle $\psi_{0}$ for varying $D_{j}, D_{c}$, and initial velocity $U_{0, X}$ (color coded in $\mathrm{m} / \mathrm{s}$ ). Dashed line is the inertial prediction $\lambda_{0}=2 \pi \mathcal{R}_{h} / \tan \psi_{0}$. (b) Theoretical helix shape including the effect of gravity with (orange curve) and without (blue curve) viscous effects for typical experimental parameters $\left(D_{c}=10 \mathrm{~mm}\right.$, $D_{j}=0.8 \mathrm{~mm}, \psi_{0}=64^{\circ}, U_{0}=2.1 \mathrm{~m} / \mathrm{s}$, and $\left.C=11\right)$. 


$$
\rho Q \frac{d(U \hat{\mathbf{t}})}{d s}=W(\tau \hat{\mathbf{t}}-\Delta P \hat{\mathbf{n}})+\rho A \mathbf{g} .
$$

In this expression, $\tau$ is the wall shear stress, while $\Delta P$ is the difference of pressure between the upper and lower sides of the jet, both averaged over the rivulet width $W(s)$ (see Supplemental Material [18]).

So far, Eq. (1) is without approximations. The inertialcapillary adhesion force is encoded in the pressure difference $\Delta P$. In the regime where a helix forms, however, $\Delta P$ will be balanced by the centrifugal acceleration along $\hat{\mathbf{n}}$, but this does not affect the shape of the helix. To estimate the wall shear stress, we assume a two-dimensional parabolic flow such that $\tau \approx-3 \eta U / h$, with $h(s)$ the rivulet thickness at the centerline. This is complemented by the geometric assumptions $A \approx W h$ and $W(s) \approx D_{j}$ (constant width) such that $\tau W=-3 C \eta D_{j}^{2} U^{2} / Q$ with $C$ a form factor encompassing the three aforementioned assumptions that we consider constant along the stream. With this, the momentum balance [Eq. (1)] takes the form (cf. Supplemental Material [18])

$$
\begin{gathered}
\frac{d U}{d s}=-\frac{48 \eta C}{\pi^{2} \rho D_{j}^{2}}\left(\frac{U}{U_{0}}\right)^{2}+\frac{g \cos \psi}{U}, \\
\frac{d \psi}{d s}=-\frac{g \sin \psi}{U^{2}}
\end{gathered}
$$

once projected along the rivulet in the unwrapped $(X, z)$ plane. The helix shape is then extracted from $\psi(s)$ as $d z / d s=\cos \psi(s)$ and $d X / d s=\sin \psi(s)$. We numerically integrate Eqs. (2) and (3) with initial conditions $\left(U_{0}, \psi_{0}\right)$ and wrap the trajectory around the cylinder to obtain the helix shape.

Figure 2(b) compares the calculated helix shape with and without viscous effects using typical experimental parameters. It clearly shows that both gravity and viscosity are necessary to quantitatively account for the experiments: The pitch increases significantly over a few turns, and the rotation slows down and eventually stops. The direct agreement with experiment is excellent (Fig. 3), with $C \sim 10$ as the only adjustable parameter that does not vary much for most of our experimental conditions. The helix shape could therefore be tuned by controlling the friction through the fluid viscosity. However, rotating the cylinder breaks the helix and coats the cylinder with a thin film.

Critical speed for helix formation. - Now that we understand the shape of the helical rivulet, we aim to describe how the jet sticks to the cylinder. In the experiment, we measure the jet deviation angle $\alpha$ with respect to the incident jet as we decrease the flow rate $Q$ from top-view pictures [Fig. 4(a)] and vary the jet size $D_{j}$, cylinder size $D_{c}$, inclination angle $\psi_{0}$, and contact angle $\theta$. Since the jet velocity is higher here than in the helix regime, we fully neglect gravitational and viscous effects. The relevant

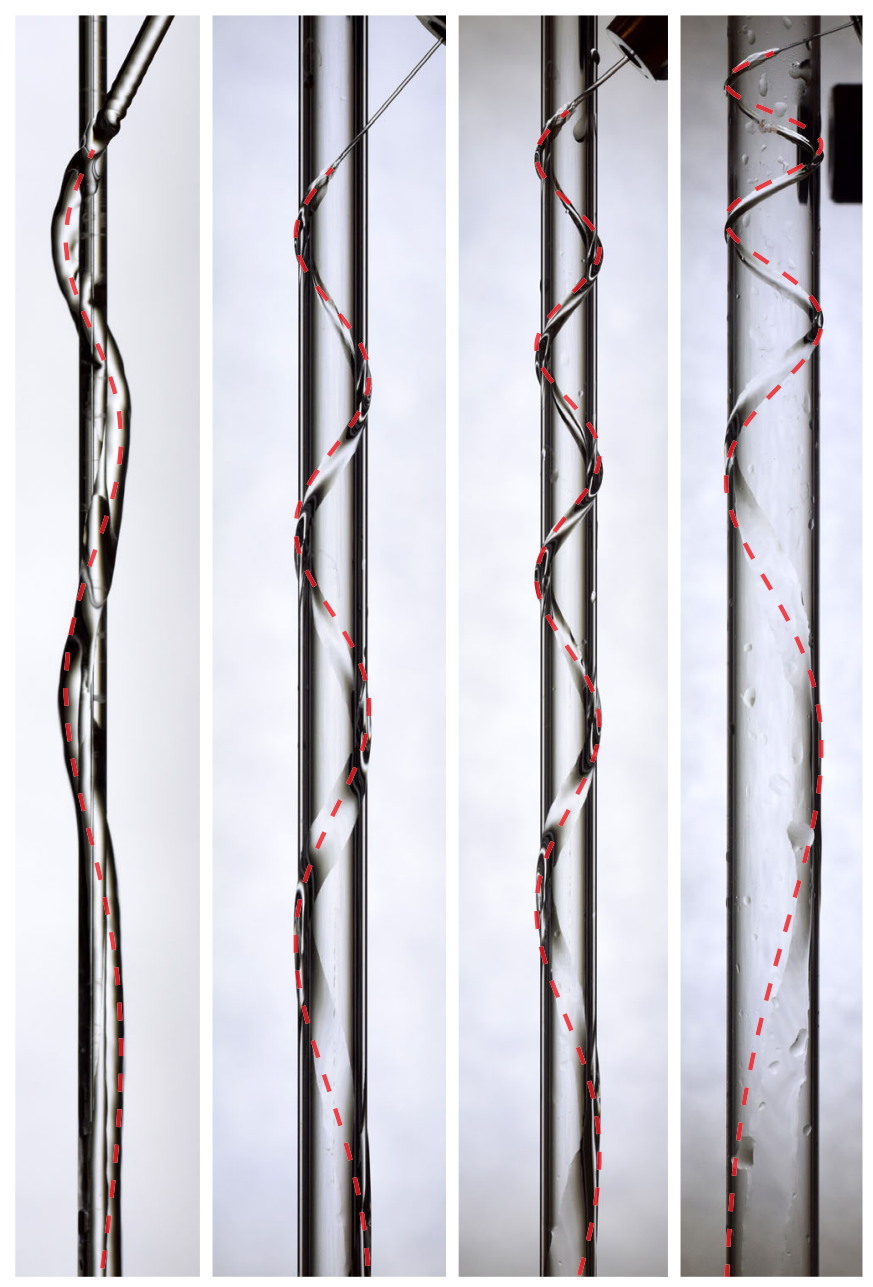

FIG. 3. Comparison between experiments and theory for a range of experimental parameters. From left to right: $D_{c}=1,5$, $5,10 \mathrm{~mm} ; D_{j}=1,0.3,0.5,0.3 \mathrm{~mm} ; \psi_{0}=26.3,40.3,47.8$, $68.6 \mathrm{deg} ; U_{0}=1.0,4.9,3.0,5.4 \mathrm{~m} / \mathrm{s} ; C=15,11.5,7,9.5$.

dimensionless numbers for the experiment are therefore the Weber number $\mathrm{We}=\rho U_{0}^{2} D_{j} / \gamma$, the dimensionless cylinder radius $\tilde{R}=D_{c} /\left(2 D_{j}\right)$, the contact angle $\theta$, and the inclination angle $\psi_{0}$.

Figure 4(c) shows $\alpha$ as a function of We for two representative dimensionless cylinder radii $\tilde{R}$ and various inclination angles $\psi_{0}$ (for these glass cylinders, $\theta=$ $32 \pm 15^{\circ}$ ). In all cases, the jet deviation is very small at high speeds $\left(\alpha \sim 5^{\circ}\right)$ and gradually increases up to a complete overturn $\left(\alpha=180^{\circ}\right)$ as the speed is decreased. Once the overturn is reached, the jet sticks to the cylinder and forms the helical rivulet. We observe that larger cylinders, smaller inclination angles, and lower contact angles result in stronger jet deviations. In fact, the dependence on $\psi_{0}$ can be scaled out by plotting the same data as a function of the Weber projected in the orthoradial direction, i.e., $\mathrm{We}_{\|}=\mathrm{We} \sin ^{2} \psi_{0}$. The collapse shown in Fig. 4(d) suggests that the problem is effectively two dimensional and can be understood from a projection in the horizontal 

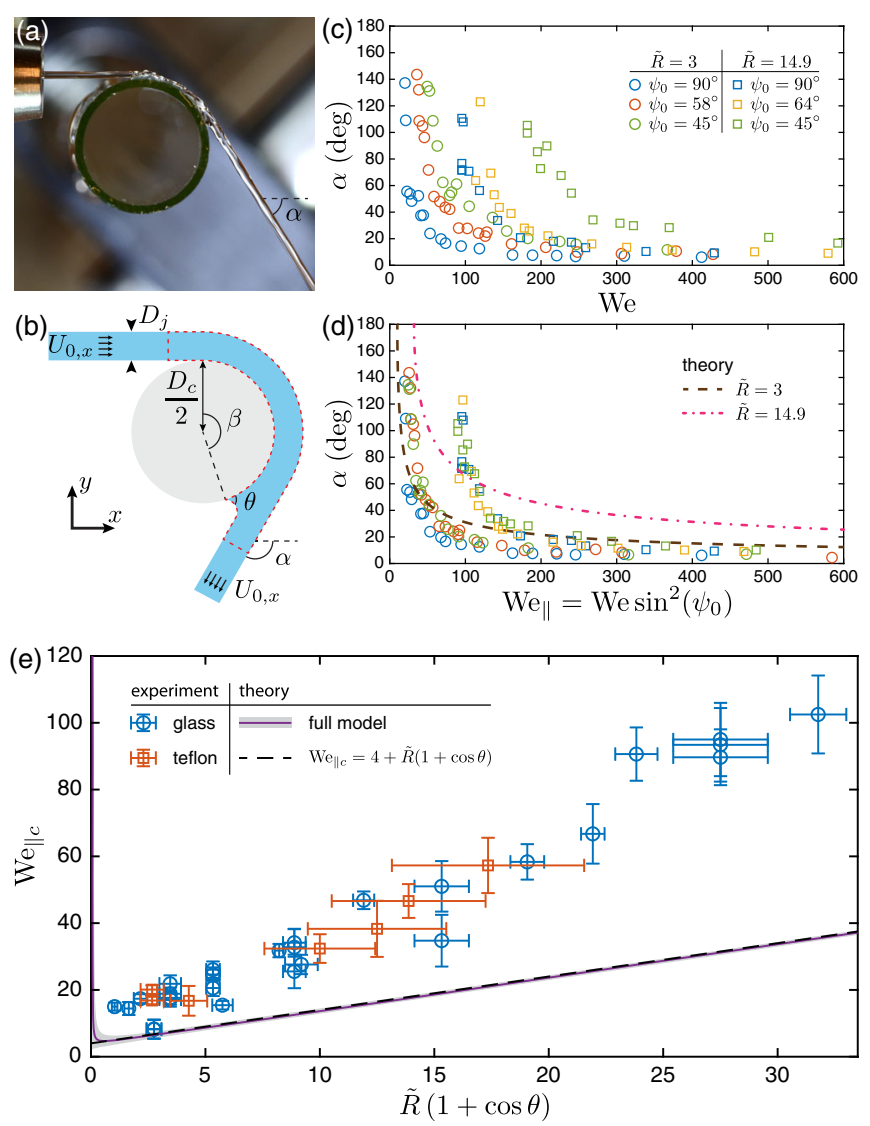

FIG. 4. (a) Top view of a typical experiment $\left(D_{j}=0.5 \mathrm{~mm}\right.$, glass cylinder $D_{c}=10 \mathrm{~mm}, Q=0.77 \mathrm{~mL} / \mathrm{s}, \quad \psi_{0}=91.5^{\circ}$ ). (b) Sketch of the 2D model defining the geometric parameters. The control volume for which we consider the momentum balance is indicated by the red dashed line. (c) Jet deviation angle $\alpha$ as a function of the Weber number We for different inclination angles $\psi_{0}$ and dimensionless cylinder radii $\tilde{R}=$ $D_{c} /\left(2 D_{j}\right)$ (glass, $\theta=32 \pm 15^{\circ}$ ). (d) Same data plotted as a function of the parallel Weber number $\mathrm{We}_{\|}=\mathrm{We} \sin ^{2}\left(\psi_{0}\right)$. Dashed lines are results from our (parameter-free) theory [Eqs. (S9) and (S10) in the Supplemental Material [18]]. (e) Critical Weber number $\mathrm{We}_{\| c}$ as a function of $\tilde{R}(1+\cos \theta)$. All experimental parameters $\left(D_{j}, D_{c}, \theta\right.$, and $\left.\psi_{0}\right)$ are varied. The purple solid curve is the full numerical solution [Eqs. (S9) and (S10) in the Supplemental Material [18] ] (the gray area represents the variations as $\theta$ is varied from $0^{\circ}$ to $179^{\circ}$ ). The black dashed line is the asymptotic expansion in the large $\tilde{R}$ limit.

plane [Fig. 4(b)]. Our experimental findings on the helix qualitatively agree with earlier experiments on fluids flowing from a solid disk [1]. Quantitatively, however, our results are different: Our experiments show that the jet can make a complete U-turn, and it is only at this point that the sticking transition happens, whereas the previous experiment put the maximum deflection at $\approx 85^{\circ}$. Another important difference is observed for the sticking transition. In Fig. 4(e), we plot the critical speed $\mathrm{We}_{\| c}$ for all our experiments and reveal a linear dependence with $\tilde{R}$. This is contrasted with the scaling $\sim \tilde{R}^{2}$ initially suggested in Ref. [1].
To rationalize these experimental results, we now develop an inertial-capillary adhesion model for the case where the jet separates from the cylinder [Figs. 4(a) and 4(b)]. We return to the momentum conservation Eq. (1) and make use of the fact that in this regime one can neglect gravity and viscosity. Consequently, both $U=U_{0}$ and $\psi=\psi_{0}$ will remain constant, as can be inferred from Eqs. (2) and (3), and therefore, $A=A_{0}$ (mass conservation). Hence, we can integrate Eq. (1) along the arc length as $\rho A_{0} U_{0}^{2}\left(\hat{\mathbf{t}}_{\text {out }}-\hat{\mathbf{t}}_{\text {in }}\right)=$ $-\int d s \Delta P W \hat{\mathbf{n}}$. This gives the momentum balance for the control volume indicated by the dashed line Fig. 4(b). Since $\hat{\mathbf{n}}$ is normal to the cylinder, it is natural to project both $\hat{\mathbf{t}}$ and $d s=d s_{\|} / \sin \psi_{0}$ onto the horizontal $(x, y)$ plane. As shown in the Supplemental Material [18], this renders the problem two dimensional based on an effective velocity $U_{0, x}=U_{0} \sin \psi_{0}$. This explains the collapse of the deviation angle $\alpha$ as a function $\mathrm{We}_{\|}$given in Fig. 4(d) and the similarity with the experiment of Ref. [1]. In the remainder, we therefore continue with a two-dimensional model and assume $A=W^{2}=D_{j}^{2}$.

To obtain a quantitative prediction for the jet deflection and the critical speed $\mathrm{We}_{\| c}$, we need to evaluate pressure $\Delta P$ on the upper and lower side of the liquid stream. The free surfaces are subjected to the Laplace pressure which can be integrated analytically along the jet. By contrast, the pressure on the solid boundary is of hydrodynamic (inertial) origin [1]: The bending of the streamlines creates a depression inside the liquid and gives rise to an adhesive force $[5,7,8]$ (sometimes called the Coandă effect). One can compute this dynamic pressure as $-\rho \int_{D_{c} / 2}^{D_{c} / 2+D_{j}}\left[u(r)^{2} / r\right] d r$ based on the velocity $u(r)$ inside the jet (with $r$ the radial coordinate). For our large cylinders $(\tilde{R} \gg 1)$, we can consider concentric circular streamlines with $u(r) \sim 1 / r$ [30]. This profile differs notably from the inviscid flow around a sharp bend $(\tilde{R} \ll 1)$ which approaches $u(r) \sim$ $r^{-1 / 2}[7,8]$, and hence, our analysis is expected to be valid only for $\tilde{R} \gtrsim 1$.

The above formulation allows a parameter-free calculation of the sticking transition (see Supplemental Material [18]) resulting in Eqs. (S9) and (S10) in the Supplemental Material [18]. Importantly, by numerically solving for the jet deviation $\alpha$, we for the first time provide a theory that captures the emergence of a minimal speed $\mathrm{We}_{\| c}$ for flow separation: The momentum balance admits two branches of solutions both observed in the experiments that annihilate through a saddle node bifurcation at $\mathrm{We}_{\| c}$ and $\alpha=180^{\circ}$, in close agreement with experiments (see Supplemental Material [18]). The prediction for $\alpha$ is plotted in Fig. 4(d) without any adjustable parameters. For small cylinders $(\tilde{R} \lesssim 5)$, the calculated deviation angles quantitatively match the experimental data, while for larger $\tilde{R}$ the agreement is only qualitative. Finally, the model resolves how the critical speed depends on all the parameters of the problem. The value of $\mathrm{We}_{\| c}$ can be computed analytically through an asymptotic expansion around the critical point 
(see Supplemental Material [18]), and for $\tilde{R} \gg 1$ it reveals that $\mathrm{We}_{\| c} \approx 4+\tilde{R}(1+\cos \theta)$. We plot in Fig. 4(e) $\mathrm{We}_{\| c}$ as a function of $\tilde{R}(1+\cos \theta)$ for all our data (different $D_{j}, D_{c}$, $\psi_{0}$, and $\theta$ ), the full numerical solutions of the model for all possible $\tilde{R}$ and $\theta$ (solid curve with gray area), and the asymptotic analytical solution (dashed line). Both the data and the full model collapse, indicating that this simple scaling is able to capture the physics of the teapot effect. Our result captures both the wettability dependence $(1+$ $\cos \theta)$ already observed [1,2], as well as the linear dependence on the solid curvature $\tilde{R}$; it therefore settles the discussion of whether the dependence of the critical speed on the radius of the jet should be quadratic (Duez et al. [1]) or linear (Dong et al. [2]). Quantitatively, the slope of the linear dependence is roughly a factor 2 off [Fig. 4(e)], which we attribute to the simplifying geometric assumptions of the jet's cross section. Calculating the full geometry of the jet goes well beyond the scope of the present contribution as we expect it to be possible only through computational fluid dynamic simulations [11].

In summary, we have studied the sticking of inertialcapillary flows to solids, also known as the teapot effect, by grazing vertical cylinders with liquid jets. We have shown that unlike in the pouring configuration, once the jet completely sticks to the solid in our setup, it forms a liquid helix whose intricate shape depends on the jet initial speed and geometry. We have then looked at the adhesion itself and how it impacts the jet when it still separates from the cylinder. Using a detailed momentum balance on the rivulet or jet, we have been able to accurately recover the observed trajectory of our liquid helices using a single fitting parameter. Moreover, we have improved the inertial-capillary adhesion scaling analysis and derived a parameter-free model that, for the first time, predicts the sticking transition and captures experimental observations semiquantitatively.

We are grateful to $\mathrm{C}$. Coulais for suggesting the equivalence with the unwrapped problem. E. J.-P. thanks Shell Global Research for funding.

[1] C. Duez, C. Ybert, C. Clanet, and L. Bocquet, Phys. Rev. Lett. 104, 084503 (2010).

[2] Z. Dong, L. Wu, J. Wang, J. Ma, and L. Jiang, Adv. Mater. 27, 1745 (2015).

[3] Z. Dong, L. Wu, N. Li, J. Ma, and L. Jiang, ACS Nano 9, 6595 (2015).
[4] M. Reiner, Phys. Today 9, No. 9, 16 (1956).

[5] J. B. Keller, J. Appl. Phys. 28, 859 (1957).

[6] S. P. Lin and M. V. G. Krishna, Phys. Fluids 21, 2367 (1978).

[7] J. VandenBroeck and J. B. Keller, Phys. Fluids 29, 3958 (1986).

[8] J. VandenBroeck and J. B. Keller, Phys. Fluids A 1, 156 (1989).

[9] S. F. Kistler and L. E. Scriven, J. Fluid Mech. 263, 19 (1994).

[10] H. Isshiki, B.-S. Yoon, and D.-J. Yum, Phys. Fluids 21, 082104 (2009).

[11] A. Kibar, Fluid Dyn. Res. 49, 015502 (2017).

[12] F. Savart, Ann. Chim. Phys. 54, 113 (1833).

[13] J. Walker, Sci. Am. 251, 144 (1984).

[14] J. Ferrand, L. Favreau, S. Joubaud, and E. Freyssingeas, Phys. Rev. Lett. 117, 248002 (2016).

[15] E. Krichtman, R. Mimon, and H. Gothait, U.S. Patent No. 8, 770, 714 (2014).

[16] Y. W. Inn, R. J. Fischer, and M. T. Shaw, Rheol. Acta 37, 573 (1998).

[17] N. M. Ribe, M. Habibi, and D. Bonn, Annu. Rev. Fluid Mech. 44, 249 (2012).

[18] See Supplementary Material at http://link.aps.org/ supplemental/10.1103/PhysRevLett.122.184501 for details on the experimental setup, derivation of the models, and indepth analysis of the inertial-capillary adhesion model, which includes Refs. [17-23].

[19] K. Birdi, D. Vu, and A. Winter, J. Phys. Chem. 93, 3702 (1989).

[20] A. Carre and M.E. R. Shanahan, J. Adhes. 49, 177 (1995).

[21] A. Lamberti, M. Quaglio, A. Sacco, M. Cocuzza, and C. Pirri, Appl. Surf. Sci. 258, 9427 (2012).

[22] T. Yasuda, T. Okuno, and H. Yasuda, Langmuir 10, 2435 (1994).

[23] W. Bouwhuis and J. H. Snoeijer, arXiv:1507.05931.

[24] J. W. M. Bush and A. E. Hasha, J. Fluid Mech. 511, 285 (1999).

[25] F. Celestini, R. Kofman, X. Noblin, and M. Pellegrin, Soft Matter 6, 5872 (2010).

[26] E. J. Watson, J. Fluid Mech. 20, 481 (1964).

[27] T. Wang, D. Faria, L. Stevens, J. Tan, J. Davidson, and D. Wilson, Chem. Eng. Sci. 102, 585 (2013).

[28] N. Le Grand-Piteira, A. Daerr, and L. Limat, Phys. Rev. Lett. 96, 254503 (2006).

[29] A. Daerr, J. Eggers, L. Limat, and N. Valade, Phys. Rev. Lett. 106, 184501 (2011).

[30] H. Lhuissier and E. Villermaux, J. Fluid Mech. 693, 508 (2012). 